\title{
Oscillatory dynamics of vasoconstriction and vasodilation identified by time-localised phase coherence
}

\author{
L W Sheppard ${ }^{1}$, V Vuksanović ${ }^{2}$, P V E McClintock ${ }^{1}$ \\ and A Stefanovska ${ }^{1}$ \\ ${ }^{1}$ Department of Physics, Lancaster University, Lancaster, LA1 4YB, UK \\ E-mail: aneta@lancaster.ac.uk \\ ${ }^{2}$ Institute of Biophysics, Faculty of Medicine, University of Belgrade, Serbia
}

\begin{abstract}
.
We apply wavelet-based time-localised phase coherence to investigate the relationship between blood flow and skin temperature, and between blood flow and instantaneous heart rate (IHR), during vasoconstriction and vasodilation provoked by local cooling or heating of the skin. A temperature-controlled metal plate $\left(\approx 10 \mathrm{~cm}^{2}\right)$ placed on the volar side of the left arm was used to provide the heating and cooling. Beneath the plate, the blood flow was measured by laser Doppler flowmetry and the adjacent skin temperature by a thermistor. Two 1-hour data sets were collected from each of 10 subjects. In each case a 30-min basal recording was followed by a step change in plate temperature, to either $24{ }^{\circ} \mathrm{C}$ or $42{ }^{\circ} \mathrm{C}$. The IHR was derived from simultaneously recorded ECG. We confirm the changes in the energy and frequency of blood flow oscillations during cooling and heating reported earlier. That is: during cooling, there was a significant decrease in the average frequency of myogenic blood flow oscillations $(p<0.05)$ and the myogenic spectral peak became more prominent; and during heating, there was a significant $(p<0.05)$ general increase in spectral energy, associated with vasodilation, except in the myogenic interval. We also show that significant $(p<0.05)$ phase coherence exists between blood flow and IHR in the respiratory and myogenic frequency intervals. Cooling did not affect this phase coherence in any of the frequency intervals, whereas heating enhanced the phase coherence in the respiratory and myogenic intervals. This can be explained by the reduction in vascular resistance produced by heating, a process where myogenic mechanisms play a key role. Weak phase coherence between temperature and blood flow was observed for unperturbed skin, but it increased in all frequency intervals as a result of heating. It was not significantly affected by cooling. We conclude that the mechanisms of vasodilation and vasoconstriction, in response to temperature change, are oscillatory in nature and are independent of central sources of variability.
\end{abstract}

PACS numbers: 02.50.Tt, 05.45.Tp, 05.10.Gg, 92.20.jm, 87.19.Hh, 05.45.Xt

Submitted to: Phys. Med. Biol. 


\section{Introduction}

Thermal regulation in humans is achieved primarily by adjustment of blood flow through the skin circulation. The peripheral circulation serves as the body's first line of the defence in the face of environmental temperature change. The second line of defence includes central neurophysiological mechanisms responsible for regulation of core temperature. These two basic control mechanisms are not independent but influence each other. It has been found that the complex mechanisms of blood flow and skin temperature regulation interact in a nonlinear way, with hysteresis and rate dependence (Kitney 1975, Vuksanović et al. 2008), suggesting that thermoregulation could usefully be studied from the point of view of the associated timescale of fluctuations. In their early work on changes in digit blood flow, Burton and Taylor (1940) attributed spontaneous frequency components below $0.1 \mathrm{~Hz}$ to thermal vasomotor control. However, the exact frequencies were not precisely established. To our knowledge, the only studies taking explicit account of the oscillatory processes that occur in both blood flow and temperature and their interactions are those by Geyer et al. (2004), Kitney (1980), Shusterman et al. (1997); and Bandrivskyy et al. (2004). The latter investigated changes in the oscillatory processes following a temperature perturbation. It yielded interesting preliminary data but suffered from two major disadvantages: first the stimulus temperature (reduced by ice-bags partially insulated from the skin with fabric) was not well-controlled, and secondly the temperature could only be reduced. In the present work a precisely-controlled thermal perturbation could be applied to a well-defined area of skin, thus ensuring that any oscillatory variability in either blood flow or recorded temperature signals is physiological in origin rather than driven by variations in the stimulus.

We applied the Morlet wavelet transform to study blood flow oscillations while the skin surface temperature was being regulated at a value associated with either maximal vasodilation or maximal vasoconstriction. However, much of the variability in skin blood flow can be attributed to central mechanisms such as the cardiac pulse pressure wave. To distinguish between the local and central influences, we determined the wavelet phase coherence between the instantaneous heart rate (IHR) and the local blood flow. The IHR was determined from an electrocardiogram (ECG).

The aim of this study was to explore rhythmical fluctuations present in blood flow time series during local perturbations of skin temperature. We sought answers to two main questions: (i) which oscillatory processes are involved in vasoconstriction and vasodilation resulting from thermal perturbations; and (ii) which of the oscillations in blood flow are of central origin and which of them are generated locally? 


\section{Background}

\subsection{Cardiovascular oscillations}

Cardiovascular system (CVS) dynamics can be modelled as a set of coupled nonlinear oscillators with time-varying frequencies, and subject to fluctuations (see for example Stefanovska and Bračič (1999), Jamšek et al. (2004), Veber et al. (2004), Smelyanskiy et al. (2005), Humeau et al. (2010)). Central oscillators integrate central neural mechanisms (Camm et al. 1996), whereas local/peripheral oscillators reflect the activity of vascular smooth muscle, sympathetic activity and, as recently demonstrated (Kvandal et al. 2006, 2003, Stefanovska et al. 1999), vascular endothelial activity. Thus several different vascular and neurophysiological processes at different levels combine to create the highly complex oscillatory patterns seen in CVS time series. Six different oscillatory components have been identified in skin blood flow (Stefanovska 2007), each corresponding to a different physiological process: (I) cardiac (0.6-2.0 Hz); (II) respiratory (0.145-0.6); (III) myogenic (0.145-0.052); (IV) neurogenic (0.021-0.052); (V) NO-dependent endothelial activity (0.0095-0.021) and (VI) NO-independent endothelial activity $(0.005-0.0095 \mathrm{~Hz})$. The activity above $0.145 \mathrm{~Hz}$ obviously originates from centrally driven processes, whereas processes with frequency below $0.145 \mathrm{~Hz}$ are expected to be of local origin (Bernjak et al. 2008, Stefanovska and Bračič 1999).

\subsection{Mechanisms of local vasodilation and vasoconstriction}

Vasoconstriction and vasodilation imply respectively the narrowing or widening of blood vessels, processes that are conventionally envisaged to occur smoothly and steadily, and monotonically in response to a given stimulus. Up to now the associated mechanisms have been investigated within this conceptual framework. It is known that vascular smooth muscle maintains an intrinsic tone (associated with vessel's radius) at body temperature (Pawlowski and Morgan 1992). The initial tone defines the extent of a stretch response, which can occur as a reaction to perturbation by a change in either flow or pressure (Folkow 1964). The vascular tonus throughout the body is also maintained at an appropriate level for thermoregulation via either global or local sympathetic mechanisms (Alvarez et al. 2006, Kellogg 2006). The global sympathetic vasodilation response to heating is dependent on cholinergic active vasodilator nerves, and the response to cooling on noradrenergic vasoconstrictor nerves. Different mechanisms are involved in the response to local temperature changes. The local vasodilatory response to heating is biphasic (Minson et al. 2001), with an initial neural component due to sensory afferent nerves, and a slower endothelium-dependent response involving the release of NO and other vasodilatory factors (Kellogg et al. 1999, Medow et al. 2008). There is also a biphasic response to local cooling, but the initial response of afferent sensory neurons acts locally on the noradrenergic vasoconstrictor nerves of the sympathetic system to initiate vasoconstriction, followed by a subsequent endothelial response to reduced NO (Hodges et al. 2006, Kellogg 2006). This homeostatic response also depends on the rate 
of cooling (Yamazaki et al. 2006), the temperature to which the skin is cooled (Johnson et al. 2005), and the duration of exposure (Nuzzaci et al. 1999). Rho Kinase has been shown to promote vasoconstriction during both phases of the response to local cooling (Thompson-Torgerson et al. 2007). Endothelium-mediated vasoactive substances are also known to act as cofactors (Kellogg 2006), and each of them may modulate the response of vascular tone to the others (Huang et al. 2000). The contribution made by the various biochemical pathways involved depends on the age of the subject. The endothelial response to increased flow, sensed as shear stress once arteriolar vasodilation begins, may also be important.

During local heating and cooling, receptors on both endothelial and smooth muscle cells respond to temperature changes to produce an appropriate redistribution of vascular resistance. Downstream of the arterioles and independently of the endothelium, venous basal tone may respond to temperature increases with constriction and vice versa (Simonet et al. 2000) to help regulate the systemic blood flow.

\subsection{Oscillations in skin temperature}

Although vasodilation and vasoconstriction are thus understood in terms of steady states, it has been known for over 70 years that, in reality, the control mechanism involves oscillations (Burton and Taylor 1940), mainly with energies below $0.1 \mathrm{~Hz}$. Spontaneous oscillatory activity of skin temperature variability below $0.1 \mathrm{~Hz}$ was found to be correlated with oscillations in blood flow, but the frequencies were not precisely resolved (Hafner et al. 2007, Shusterman et al. 1997).

Skin temperature oscillations have been particularly observed during temperature provocation. Entrained oscillations in skin blood flow in the fingers have also been demonstrated when periodic thermal stimuli (periods of 20 and $80 \mathrm{~s}$ ) are applied to the contralateral hand (Kitney 1974, 1975). These responses could indicate global thermoregulatory activity coordinated by the sympathetic nervous system rather than the local thermoregulatory response. Because skin heating causes vasodilation, it increases average blood flow. It may have an effect on blood flow variability in all frequency intervals. Geyer et al. (2004) found an increase in the proportion of signal energy in the endothelial related interval and a relative decrease in the myogenic interval.

\subsection{Oscillations in instantaneous heart rate}

The heart drives blood flow through the body, and changes in heart rate affect blood pressure and local perfusion. The heart rate is known to oscillate in time with respiration (well-known as respiratory sinus arrhythmia, or RSA) (Camm et al. 1996). Frequency analysis reveals a spectral peak at around $0.1 \mathrm{~Hz}$ which may be associated with so-called Mayer waves in systemic blood pressure (Eckberg 2010) and skin blood flow variability in the myogenic interval. Low frequency variability in IHR is found to occur in the same spectral intervals as those identified in blood flow (Kvandal et al. 2006, Shiogai et al. 2010, Stefanovska 2007) using the Morlet wavelet transform. 


\section{Mathematical and experimental methods}

\subsection{The Morlet wavelet transform and wavelet phase coherence}

In order to capture the time variability of the basic (characteristic) oscillation frequencies in blood perfusion signals, the Morlet wavelet transform was applied to data (Stefanovska and Bračič 1999, Stefanovska et al. 1999) recorded as described below. The transform is obtained by convolving the signal with the Morlet wavelet, a complex plane wave of centre frequency $f_{0}$ multiplied by a Gaussian envelope of unit width. A family of similar wavelets is generated at a range of scale values, $\sigma$, separated by percentage increments appropriate to the frequency resolution of the wavelet. The wavelets are normalised by spectral energy:

$$
\psi(\sigma, t)=\frac{1}{\sigma^{\frac{1}{2}}} e^{\frac{-i 2 \pi f_{0} t}{\sigma}} e^{\frac{-t^{2}}{2 \sigma^{2}}}
$$

where $t$ is time. The frequencies of the wavelets are inversely proportional to their scales. The lowest frequency component that can reliably be detected depends on $f_{0}$ and the length of the available time-series. The wavelet transform of a time-series $x(t)$ can be written as

$$
W(\sigma, t)=\int_{-\infty}^{\infty} \psi(\sigma,(t-\tau)) x(\tau) d \tau
$$

where the integration is taken over the dummy variable $\tau$. $W(\sigma, t)$ is the convolution of the complex wavelet with the time-series at each scale, giving a matrix of complex values that define an absolute amplitude and relative phase at each point in time for each frequency component; note that, in practice, the operation is discretised in both time and frequency. In the frequency domain the wavelet acts as a filter with a Gaussian profile, centred at $f_{0} / \sigma$, with a width inversely proportional to the scale. The phase of the wavelet transform at each point in time is equivalent to the Hilbert phase of the filtered signal.

The wavelet defined in (1) is truncated where the magnitude of the envelope is sufficiently small. Where its edges overhang the edges of the signal, the reliability of the calculated $W(f, t)$ is reduced, so the wavelet transform is also cut to remove values which are too close to the edge. This factor limits the lowest frequency that can reliably be identified in the transform.

Where two noisy signals with different spectra include power at the same frequency the question arises as to whether the oscillations are due to related or unrelated causes. The method of phase coherence enables us to determine whether the oscillations detected are significantly correlated over time. For a given frequency component, the phases $\left(\phi_{1}\right.$ and $\left.\phi_{2}\right)$ are determined at each point in time and the phase difference between the two signals is then calculated. If each phase difference is considered as a unit phasor, the amplitude of the mean phasor is the phase coherence

$$
\Pi(\sigma)=\frac{1}{n}\left|\sum_{t=1}^{n} e^{i\left(\phi_{1}(\sigma, t)-\phi_{2}(\sigma, t)\right)}\right| .
$$


The definition is equivalent to that used by Bandrivskyy et al. (2004). The magnitude of the phase coherence thus expresses the tendency of the phase difference between signals to remain fixed over time (e.g. to remain at 0 or some other fixed phase delay). The more similar the various phase difference values are, the greater the size of the total (or mean) phasor. If no particular phase difference is preferred, the total phasor will be small, like a random walk in the complex plane.

However, even unrelated signals will have a finite phase coherence over a finite period of time. That of two uncorrelated signals is expected to fall off as $n^{-1 / 2}$, where $n$ is the number of independent samples of the phase difference as it varies in time. The wavelet phase increases monotonically, defined within $-\pi<\phi \leq+\pi$. The wavelet transform phase grows at a rate equivalent to an instantaneous frequency for that component. If phase growth between two different signal transform components of the same frequency was purely linear (no variability), then all samples are equivalent and any (possibly arbitrary) phase difference between the time-series would be preserved throughout the signal, making the phase coherence trivially equal to unity. In fact the growth of phase for each frequency component is nonlinear for all physiological processes, and it is this nonlinear growth of phase that enables independent measurements of the phase difference between two phase time-series. The longer the autocorrelation time of each signal, the less independent can be the values of the phase difference time-series, and thus the higher the phase coherence of even unrelated signals. The latter tends to increase towards unity for the lowest frequencies in the wavelet transform, as the corresponding number of cycles in the time-series decreases.

To estimate a distribution of phase coherence functions for unrelated signals, and thus gauge the significance of the phase coherence at each frequency, we use the method of surrogates. Where several bivariate data sets are available, we calculate the wavelet phase coherence of every possible mismatched pair of signals, which by construction are unrelated. The actual value of phase coherence obtained at each frequency can then be compared with the mean, plus one and plus two standard deviations of this surrogate distribution. Furthermore, the distribution of actual phase coherence values for each bivariate data set can be compared under different temperature conditions.

\subsection{Wavelet spectral normalisation}

The interpretation and comparison of wavelet transform plots and wavelet amplitude and energy spectra is not possible without understanding the scale-dependent normalisation of a wavelet, associated with the variable width of the frequency increment of which each value is representative. Normalisation must be appropriate to the area in timefrequency space represented by each calculated value of the transform, and the graphical presentation of these values must be appropriate to allow comparison of the spectral features at different frequencies.

For the wavelet normalisation given in Eq. (1), we calculate the wavelet energy 
terms as

$$
E=\sum_{t} \sum_{\sigma} W(\sigma, t) W^{\star}(\sigma, t)\left(f\left(\sigma_{n-1}\right)-f\left(\sigma_{n+1}\right)\right) / 2 .
$$

where the asterisk denotes complex conjugate.

\subsection{Data recording and analysis}

Subjects 10 healthy subjects ( 7 males and 3 females; mean age (30.8 \pm 3.0 ) years) participated in the study. They each lay supine on a bed in a well-ventilated room at $20.5 \pm 1.0^{\circ} \mathrm{C}$ and relaxed for 20 minutes prior to the measurements. A basal measurement of 30 minutes length was made with the (inert) temperature control plate in situ, followed by a 30 minute recording in which the temperature of the plate was controlled at $24.0 \pm 0.1^{\circ} \mathrm{C}$. After 20 minutes resting with the thermal control plate removed, it was replaced for a second basal recording of 30 minutes, followed by a 30 minute recording in which the control surface was instead heated to $42.0 \pm 0.1^{\circ} \mathrm{C}$. The basal recordings minimised problems of reproducibility (Roustit et al. 2010) associated with the replacement of the sensor after the resting period. Temperature values were chosen to produce a maximal response (Kellogg 2006), but the stimulus was applied over a small area to avoid provoking central thermoregulatory mechanisms (verified by the simultaneous control measurements).

All participants gave their informed consent in writing. The investigation conformed with the principles outlined in the Declaration of Helsinki and was approved by the Local Ethics Committee.

Data recording procedure Skin temperature was locally perturbed by contact with a copper plate. The dimensions of the plate were $32 \times 38 \mathrm{~mm}\left(\approx 10 \mathrm{~cm}^{2}\right.$ area $)$. The weight was $1.5 \mathrm{~N}$. Thus the excess pressure was $1.2 \mathrm{kPa}$ (less than $10 \%$ of blood pressure). As shown in figure 1 , the plate was placed on the volar aspect of the lower arm, with a small aperture $\left(\approx 8 \mathrm{~mm}^{2}\right)$ in the centre to accommodate the laser-Doppler flowmetry probe. A temperature probe was placed under the plate with its thermally insulated surface in contact with the plate and its conductive surface in contact with the skin. Peltier elements linked to the plate and to the temperature controller (Temperature Controller, MTTC-1410, Melcor Corp., Trenton NJ, USA) brought the temperature of the plate to within $0.1{ }^{\circ} \mathrm{C}$ of the target temperature within a few seconds. The temperature sensor (Thermilinear, YSI Inc., Yellow Springs OH, USA) and associated signal conditioning unit (Cardiosignals, Jožef Stefan Institute, Ljubljana, Slovenia) enabled skin temperature to be recorded with a resolution of $0.0003{ }^{\circ} \mathrm{C}$ between 20 and $40{ }^{\circ} \mathrm{C}$, using 22-bit A/D conversion scaled to $\pm 5 \mathrm{~V}$. A second identical set of blood flow and temperature sensors was placed $\approx 10$ centimetres lower down on the same arm to provide simultaneous control measurements on unstimulated skin. In every case an area of skin free from large vessels was selected. The distance between the LDF probe and corresponding temperature sensor was minimized at both locations ( $\approx 1$ centimetre). 


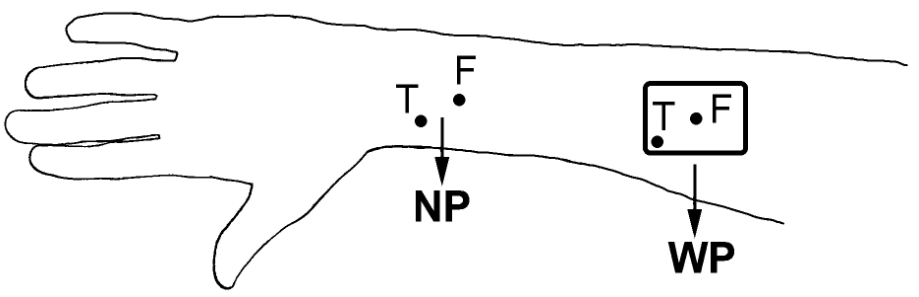

Figure 1. Sketch showing sensor placement on the subject's left arm which rested comfortably, palm up, alongside the (supine) body. The flow (F) and temperature (T) measurements were made as close together as possible, with the temperature control plate (WP) covering both sensors. In addition to the control measurements made before the plate temperature was set, a second set of sensors was placed further along the arm with no plate (NP) for simultaneous control measurements before and during heating and cooling. All sensors were placed so as to avoid obvious veins, scars and hair.

The laser-Doppler probe (DP1T-V2) was connected to a DRT4 unit (Moor Instruments, Axminster, Devon, UK), enabling cutaneous blood flow to be recorded in standard arbitrary perfusion units (AU), proportional to the density and velocity of red blood cells (Leahy et al. 1999, Nilsson et al. 1980). The fibre separation in the Moor Instruments (Axminster UK) laser Doppler DP1T-V2 probe is $0.5 \mathrm{~mm}$. The signal sampling rate was $1200 \mathrm{~Hz}$, re-sampled to $10 \mathrm{~Hz}$ for the temperature and blood flow signals prior to further analysis. A continuous three-point ECG was recorded simultaneously using the same signal conditioning unit. The voltage applied to the Peltier elements by the controller was determined by the bulk plate temperature (measured independently of the skin surface temperature recordings) relative to the set temperature. The control response was damped to avoid introducing spontaneous oscillatory responses. The spectrum of spontaneous fluctuations was checked with the device attached to a wooden block to verify that there were no spurious spectral peaks in the bands of physiological interest.

Detection of instantaneous heart rate (IHR) and data pre-processing The IHR was calculated with resolution determined by the sampling frequency of $1200 \mathrm{~Hz}$. In between the R-peaks, the phase was determined by linear interpolation. The skin temperature and LDF time-series were detrended by subtraction of a moving average over 400 seconds to remove the trend and any variability below $0.0025 \mathrm{~Hz}$. In $11 \%$ of recordings, movement artefacts in the signals were removed. Some disturbances of a few seconds duration were replaced by interpolated values, and disturbances at the ends of some pre-heating signals were removed by truncation of up to $200 \mathrm{~s}$ of data. All signals were divided at the point of temperature perturbation and the time-series before and during the cooling or heating were analysed separately using the continuous Morlet wavelet transform. Control measurements were analysed in the same way. 
Estimation of wavelet spectral energy A Morlet mother wavelet with $f_{0}=1$ was generated, with a family of wavelets re-scaled by successive increments of $5 \%$. The highest frequency wavelet was at $2.5 \mathrm{~Hz}$ to include the cardiac oscillations and the lowest at $0.0025 \mathrm{~Hz}$, limited by the length of the signal. Represented in the time domain, the wavelets were truncated at the point where the size of the Gaussian wavelet envelope dropped below 0.001. Transform values were removed where the size of the wavelet envelope overhanging the edge of the time-series was more than 10 percent of its peak value. The wavelet energy spectrum was determined and then integrated over each of the six frequency bands using the appropriate frequency increment.

Estimation of wavelet phase coherence The phase coherence was determined from the phase values of blood flow and temperature, and blood flow and IHR; in each case the phase was calculated by use of the wavelet transform. A distribution of spurious phase coherence functions was generated by mismatching either blood flow and temperature, or blood flow and IHR, wavelet phase series from the different subjects. A mean phase coherence level more than two standard deviations above the mean of a group from the surrogate distribution was deemed to indicate a non-spurious relationship between the signals at that frequency for that subject. Additionally, The distribution of actual phase coherence values at each frequency for all subjects was compared with the surrogate distribution using the Kolmogorov-Smirnov rank sum test.

Statistical tests By use of a paired Wilcoxon signed rank test, changes in the distribution of wavelet energy spectra during the temperature perturbation were tested against a null hypothesis: viz that the median change for all subjects in energy in a given band, or in terms of total spectral energy, was zero. To compare phase coherence values before and after a perturbation, a paired Wilcoxon signed rank test was used to determine whether the median difference in phase coherence values for each subject measured before and during the perturbation was significant. The Kolmogorov-Smirnov rank sum test was also applied to check for significant changes in the distributions of energy or phase coherence values.

\section{Results}

Representative examples of skin blood flow and skin temperature measurements are shown in figure 2, accompanied by wavelet transforms of the blood flow signal during local skin cooling (a) and heating (b). The well-known initial vasodilation biphasic response to heating (Minson et al. 2001) shown in the middle graph of figure 2(b) is not directly related to the oscillatory behaviour on which we focus, so the first 300 seconds of the response to heating was excised prior to further analysis.

Group results for skin blood flow and temperature, and corresponding total spectral energies of the wavelet transform of both signals, are presented as median values and box plots in figure 3 , with the corresponding statistical analyses in table 1 . As can be 

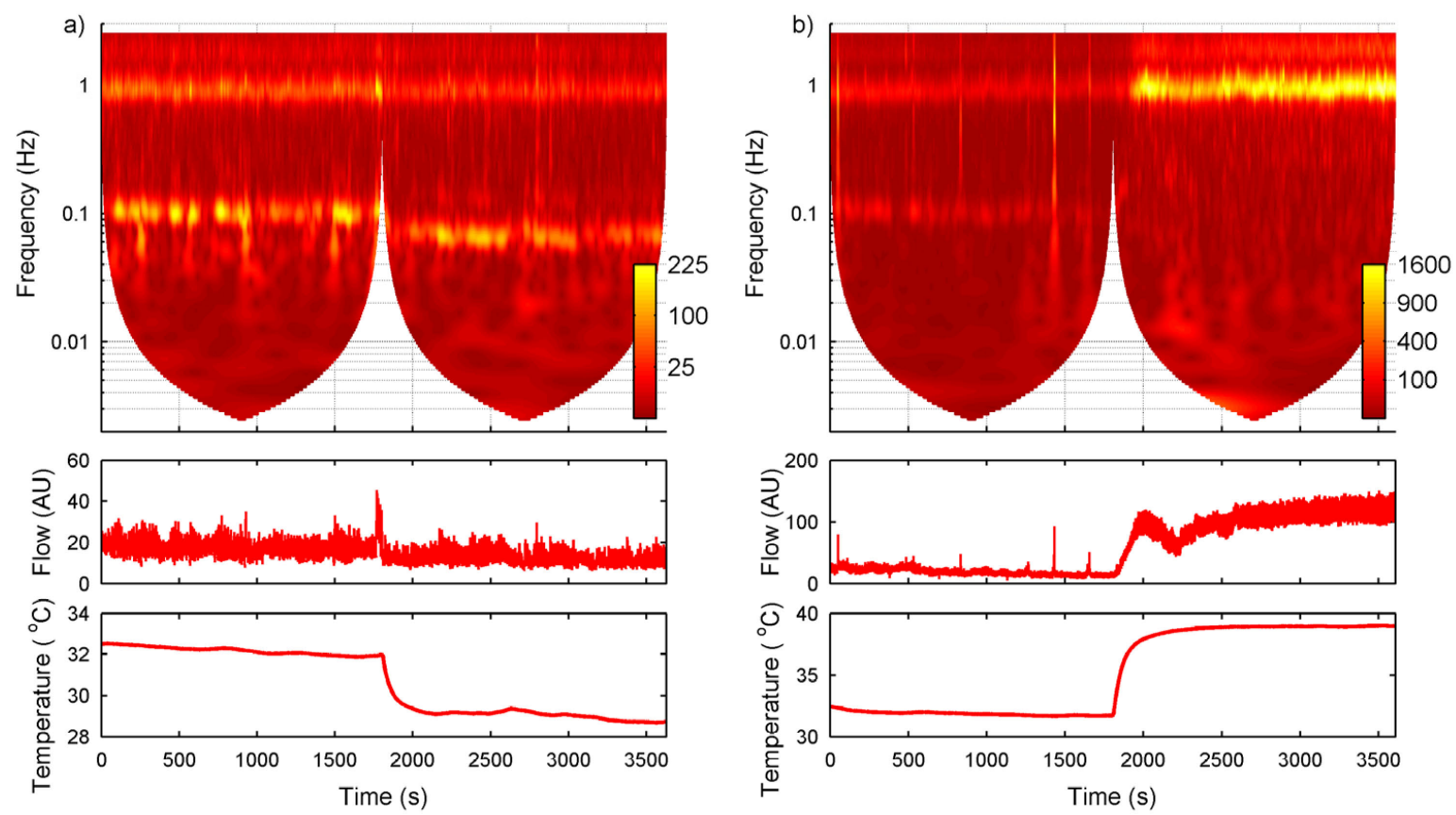

Figure 2. Blood flow wavelet transforms, raw flow signal and recorded skin temperature under the temperature regulation plate over 30 minutes of measurements without temperature perturbation, followed by 30 minutes with the plate temperature set (a) to $24{ }^{\circ} \mathrm{C}$ and (b) to $42{ }^{\circ} \mathrm{C}$.

seen, the mean values and the total energy of variability in blood flow and temperature were different from person to person.

The mean IHR was $0.97 \pm 0.13 \mathrm{~Hz}$ and $0.97 \pm 0.07 \mathrm{~Hz}$ before cooling and and heating respectively, and underwent no significant changes during the course of the protocol.

\begin{tabular}{|lr||l|l|l|l|l|l|l|l|}
\hline \multicolumn{2}{|c|}{ Unpaired } & $(\mathrm{a})$ & $(\mathrm{b})$ & $(\mathrm{c})$ & $(\mathrm{d})$ & $(\mathrm{e})$ & $(\mathrm{f})$ & $(\mathrm{g})$ & $(\mathrm{h})$ \\
\hline$p$ & $(\mathrm{NP})$ & 0.9748 & 0.9748 & 0.6751 & 0.9748 & 0.0310 & 0.3129 & 0.6751 & 0.1108 \\
$p$ & $(\mathrm{WP})$ & 0.3129 & 0.9748 & 0.0012 & 0.0310 & 0.0000 & 0.0002 & 0.0000 & 0.1108 \\
\hline
\end{tabular}

\begin{tabular}{|ll||l|l|l|l|l|l|l|l|}
\hline \multicolumn{2}{|l|}{ Paired } & $(\mathrm{a})$ & $(\mathrm{b})$ & $(\mathrm{c})$ & $(\mathrm{d})$ & $(\mathrm{e})$ & $(\mathrm{f})$ & $(\mathrm{g})$ & $(\mathrm{h})$ \\
\hline$p$ & $(\mathrm{NP})$ & 0.4553 & 0.3562 & 0.0010 & 0.8743 & 0.0066 & 0.1194 & 0.1233 & 0.0325 \\
$p$ & $(\mathrm{WP})$ & 0.9900 & 0.4475 & 0.0000 & 0.0098 & 0.0000 & 0.0115 & 0.0000 & 0.7762 \\
\hline
\end{tabular}

Table 1. Statistical analyses of the data shown in figure 3. The $p$ values from unpaired and paired tests are given for each panel of the figure, both for no plate (NP) and with the plate $(\mathrm{WP})$. Values below $p=0.05$ are italicised to indicate their significance. There is no difference in the results of the two tests except for cases (c) and (h) which suggest that there might perhaps be some collateral effect (Kitney 1974, 1975). 

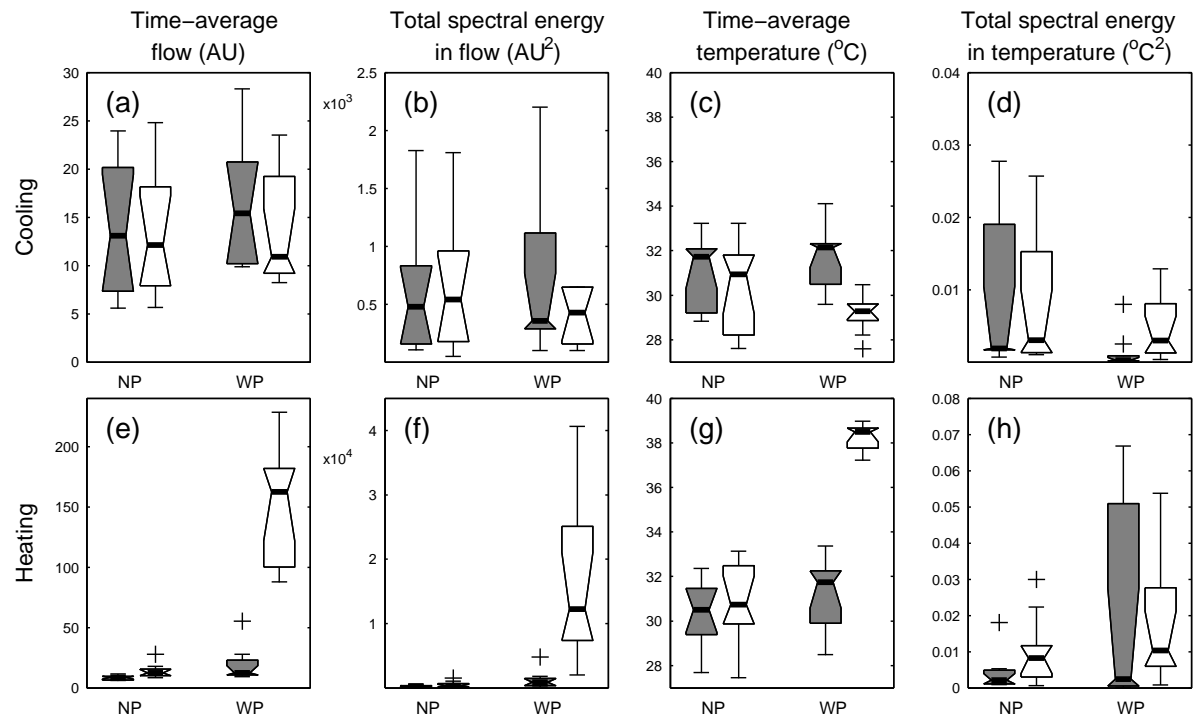

Figure 3. Boxplots for measurements made away from the temperature control plate (NP), and under the temperature control plate (WP), before (grey), and after (white) the temperature of the plate is set. Top row, for cooling: (a) average flow; (b) total wavelet energy of variability in flow between 0.005 and $2 \mathrm{~Hz}$; (c) average temperature; and (d) total wavelet energy of variability in temperature for all six intervals between 0.005 and $2 \mathrm{~Hz}$. Bottom row, for heating: (e) average flow; (f) total wavelet energy of variability in flow between 0.005 and $2 \mathrm{~Hz} ;(\mathrm{g})$ average temperature; and (h) total wavelet energy of variability in temperature for all six intervals between 0.005 and 2 $\mathrm{Hz}$.

Cooling The cooled plate resulted in a significant mean skin temperature change from $31.7 \pm 1.5$ to $29.1 \pm 0.8^{\circ} \mathrm{C}(p<0.01)$. During cooling, one subject underwent a temporary vasodilation response. When this subject was excluded from the analysis a significant drop in mean flow from 16.3 \pm 7.0 to $12.6 \pm 5.3 \mathrm{AU}$ was observed. The total energy in the flow spectrum does not change significantly with cooling $(p>0.05)$, but does change significantly with heating $(p<0.05)$. Figures $3(\mathrm{~b})$ and 3(f) show the different responses of spectral energy in flow to cooling and heating. The total energy of variability in temperature was different from person to person. The set of temperature recordings made under the plate before cooling had lower variability than simultaneous control measurements, but when cooling began the signal energy in all intervals increased to levels comparable with the control measurements (Figure $3 \mathrm{~d}$ ).

Heating The heated plate resulted in a significant rise in mean skin temperature from $31.1 \pm 1.5$ to $38.2 \pm 0.6{ }^{\circ} \mathrm{C}$ and a significant mean blood flow increase from $19.1 \pm 14.1$ to $152.3 \pm 46.0 \mathrm{AU}$, indicating pronounced vasodilation as expected. The total energy in the flow spectrum changes significantly with heating $(p<0.05)$. 

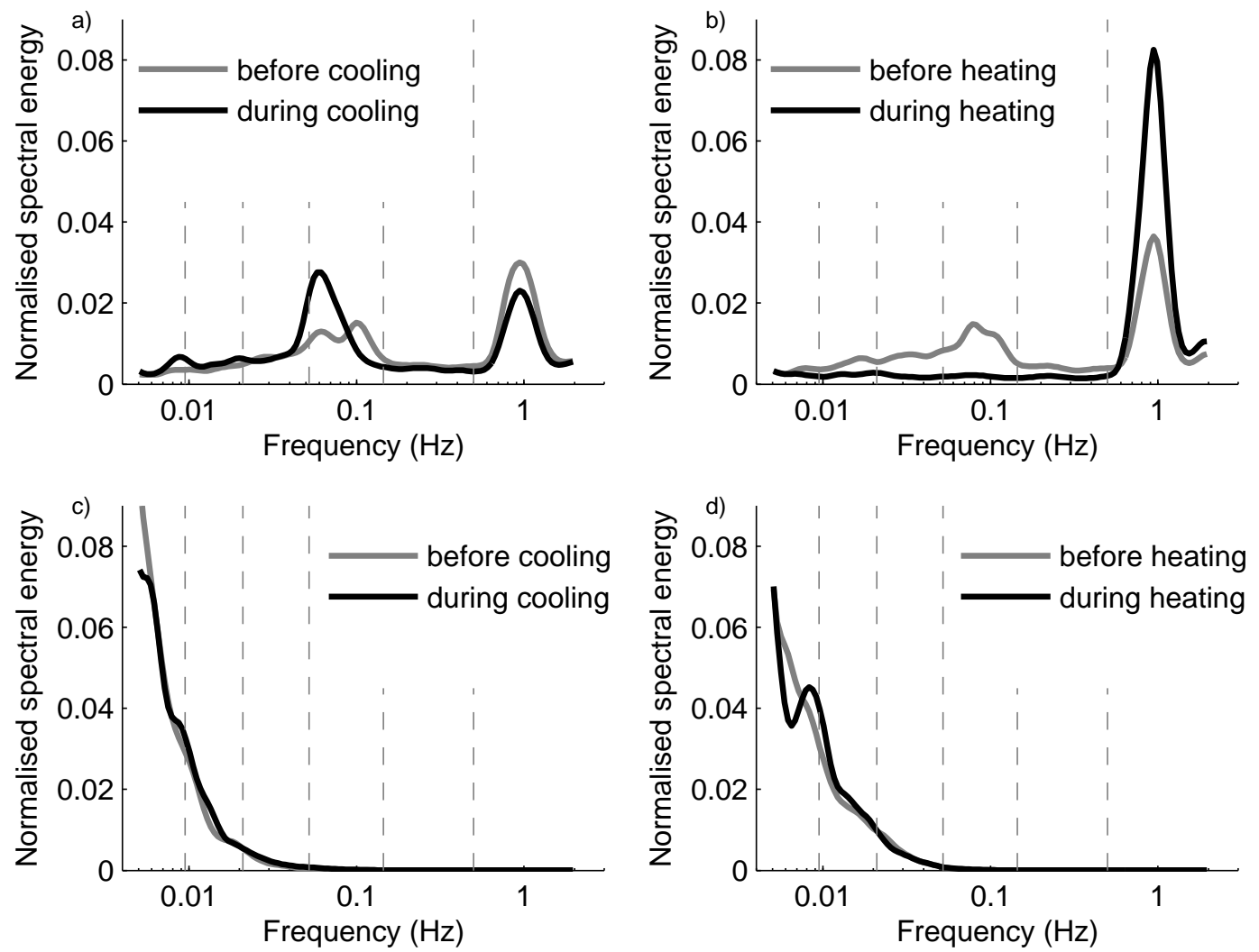

Figure 4. (a) Normalised wavelet energy spectra of blood flow before and during cooling, and (b) before and during heating. (c) Normalised wavelet energy spectra of skin temperature, recorded under the temperature regulation plate before and during cooling, and (d) before and during heating. In each case, the vertical dashed lines indicate the boundaries of frequency intervals I - VI.

\subsection{Myogenic frequency decreases with cooling}

The most significant change in the frequencies of the maxima in energy spectra compared to the control measurements during either cooling or heating was a decrease in the frequency of the spectral peak in band III as a result of cooling, associated with myogenic activity as shown in figure 2(a). This is in agreement with the earlier report by Bandrivskyy et al. (2004). The decrease is also apparent in the wavelet transform spectra obtained by averaging the data from all subjects, as shown in figure 4(a). The median myogenic frequencies before and during cooling were $0.084 \mathrm{~Hz}$ and $0.060 \mathrm{~Hz}$, respectively. The distributions of frequency values obtained were significantly different $(p<0.05)$. Note that during cooling the myogenic peak falls on the boundary between the myogenic and neurogenic frequency bands, and becomes much more sharply defined.

\subsection{Spectral energy changes in heating and cooling}

Cooling The energy found at the myogenic peak was higher in all ten subjects during cooling. The normalised spectral energy within the myogenic frequency band increased 

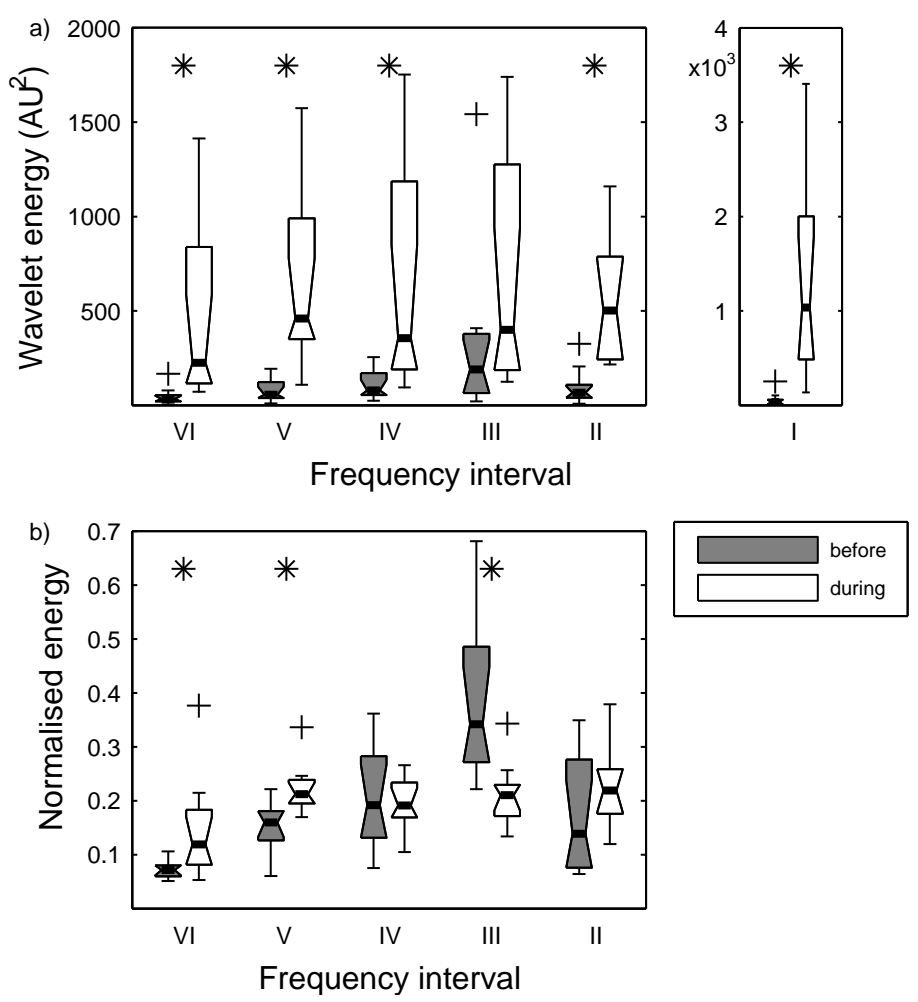

Figure 5. (a) Boxplots of absolute wavelet energy of blood flow variability in each frequency interval before heating and with heating. The cardiac interval is shown separately to accommodate the high energies after heating. (b) Boxplots showing wavelet energy of blood flow variability in each frequency interval before heating and with heating, normalised by the total energy in five of the six intervals, excluding the cardiac frequency interval. Significant changes $(p<0.05)$ are indicated by a star.

during cooling and this was statistically significant when compared with the data obtained from the control site either before or during cooling $(p<0.05)$. At the same time the normalised spectral energy within the cardiac interval decreased. The spectral peak associated with oscillations in the endothelial interval VI became more pronounced as shown in figure 4(a), but the associated change in energy was not statistically significant.

Heating There is an overall increase in the total spectral energy of blood flow during heating, together with a significant absolute increase $(p<0.05)$ in all six intervals except the myogenic one (figure 5(a)). The most pronounced increase in energy is that of the cardiac component, on account of the reduced resistance caused by vasodilation. This trivially results in a reduction of the proportion of total signal energy attributable to each of the other intervals. Excluding the cardiac interval, and normalizing instead by the total energy in intervals II to VI, we find a significant reduction in the proportion of signal energy in the myogenic interval (III), and a significant increase in the proportion found in the endothelial intervals V and VI as shown in figure 5(b). These results apply 

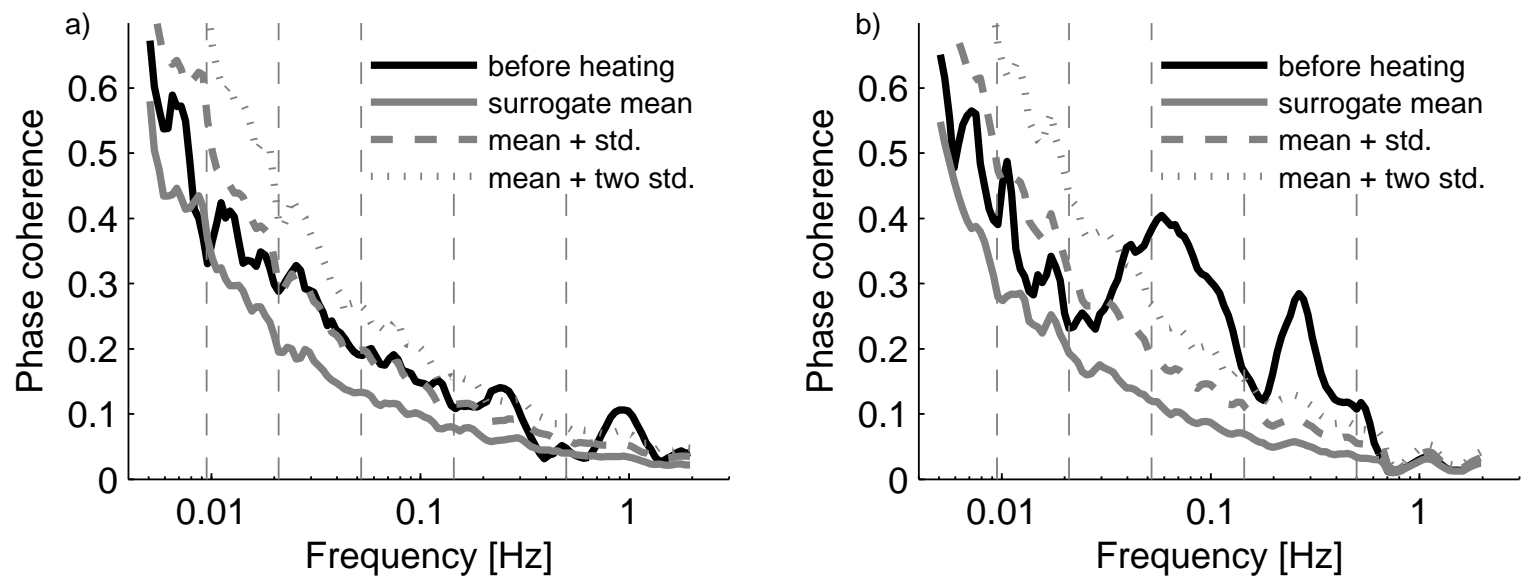

Figure 6. a) Wavelet phase coherence of skin temperature and blood flow variability data compared with the distribution of spurious phase coherence values. For each subject, the actual phase coherence was calculated and so were the mean and standard deviation of mismatch surrogate phase coherence values. The averages over all subjects (actual, surrogate mean and surrogate standard deviation) are plotted here. b) Wavelet phase coherence of IHR and blood flow variability data compared with the distribution of spurious phase coherence values. For each subject, the actual phase coherence was calculated and so was the mean and standard deviation of mismatch surrogate phase coherence values. The average over all subjects (actual, surrogate mean and surrogate standard deviation) are plotted here. In each case, the vertical dashed lines indicate the boundaries of frequency intervals I - VI.

to all three comparisons: normalised energies during heating compared to either values under the plate, or on the control site, before or during heating.

\subsection{Temperature oscillations}

Cooling Analyses of temperature oscillations for heating and cooling are shown in figure 4(c). During cooling a statistically significant increase in absolute values occurred in intervals $\mathrm{V}(p<0.0005)$ and VI $(p<0.05)$. When the data are normalised the increase becomes less pronounced ( $p=0.05$ and $p=0.7$ respectively).

Heating During heating, a pronounced peak appears in interval VI as shown in figure $4(\mathrm{~d})$, but the increase is not statistically significant.

\subsection{Phase coherence of blood flow and skin temperature}

We calculated the wavelet phase coherence between skin temperature and the laser Doppler signal, before and during heating and cooling, both for the temperatureperturbed site and for the control site. It is interesting to note that statistically significant phase coherence (more than two standard deviations above the values obtained from mismatched surrogates) exists in the interval $0.052-2.0 \mathrm{~Hz}$ in the 
temperature-unperturbed state. This result was obtained from signals measured beneath the plate as well as from signals measured on the control site. The mean (for all subjects) phase coherence obtained from signals recorded under the plate before heating is shown in figure 6(a). We comment that the phase coherence found under the plate when the Peltier elements were inactive was generally slightly higher than that for the control site.

Cooling When a paired Wilcoxon signed rank test was applied to compare the phase coherence values obtained during cooling, and the pre-cooling distributions, significant differences $(p<0.05)$ were obtained in localised frequency ranges within intervals I, III, V and VI, as shown by the shading and stars in figure 7(a). In interval III the coherence decreased, probably on account of the decreased myogenic frequency, whereas in intervals I, V and VI the coherence increased.

Heating As can be seen from 7(b), a marked increase of phase coherence occurred on heating. Applying a paired Wilcoxon signed rank test showed that there was a statistically significant $(p<0.05)$ increase in all intervals as indicated by the shading and stars. This significance arises for all three comparisons of phase coherence: (i) between the heated place before and during heating; (ii) between the heated place and the control site before heating; and (iii) between the heated place during heating and the control site.

\subsection{Phase coherence of blood flow and IHR}

We evaluated the phase coherence between blood flow and IHR in three different ways, by comparing values obtained with: (i) blood flow from beneath the plate with the Peltier elements switched off; (ii) from the simultaneously recorded blood flow on the control site; and (iii) from the simultaneously recorded blood flow on the control site when the Peltier elements were active. The mean (for all subjects) pre-heating phase coherences of IHR and blood flow under the plate are shown in figure 6(b). This figure represents the basal state, which remained unchanged at the control site during cooling, before heating and during heating. We observe clear peaks in phase coherence at the respiratory and myogenic frequencies, well above the two-standard-deviation significance threshold of the surrogate distribution.

Cooling The phase coherence between IHR and blood flow under the plate decreased in the myogenic interval during cooling, as shown by the shading and stars in figure figure $7(\mathrm{c})$. This was on account of the decreased frequency of myogenic oscillations, as seen in the blood flow and discussed above.

Heating During heating the phase coherence was found to increase in the respiratory and myogenic bands as shown by the shading and stars in figure $7(\mathrm{~d})$. 
a)
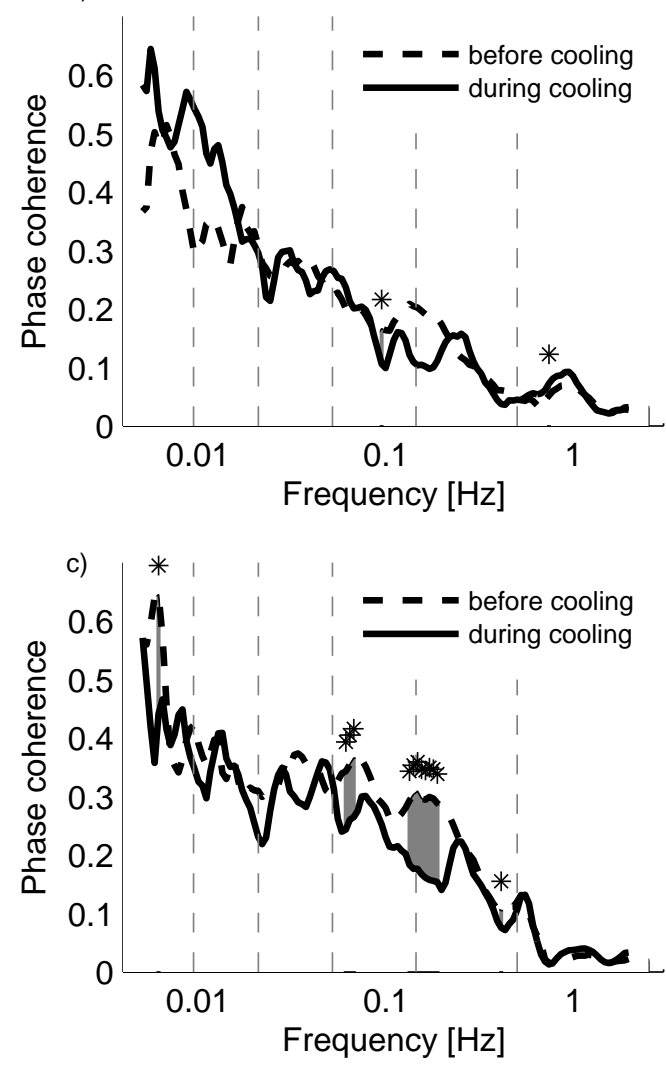

b)

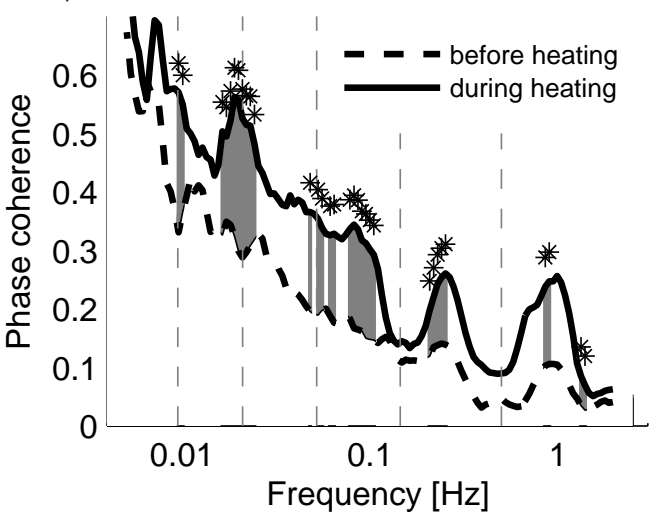

d)

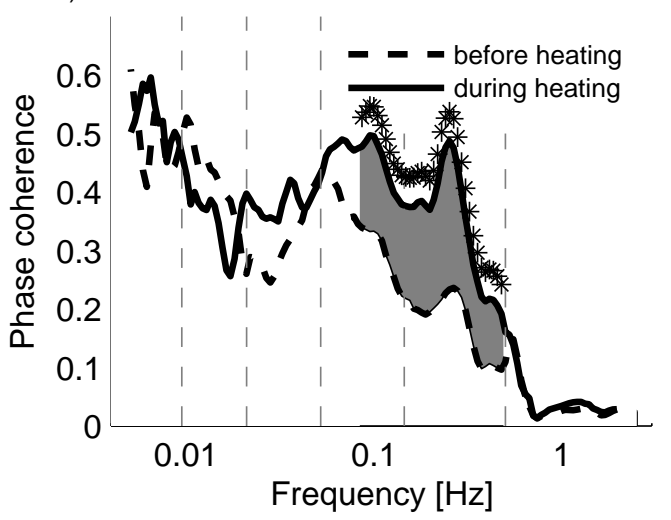

Figure 7. (a) Mean wavelet phase coherence of variability in temperature and blood flow before and during cooling, and (b) before and during heating. (c) Mean wavelet phase coherence of variability in IHR and blood flow before and during cooling, and (d) before and during heating. In each case, shading and stars are used to indicate parameter ranges where the differences were statistically significant $(p<0.05)$. In each case, the vertical dashed lines indicate the boundaries of frequency intervals I - VI.

\section{Discussion}

Local heating or cooling of the skin results in local vasodilation or vasoconstriction of the vasculature. The results presented above provide detailed insight into the associated myogenic oscillatory activity and show that it is central to both processes: in vasoconstriction there is increased myogenic activity, involving increased amplitude of oscillations, decreased frequency, and decreased average vessel radius. In vasodilation, myogenic activity is dramatically decreased. This reduction is associated with increased vessel average radius, decreased resistance, and greatly increased average blood flow. In addition, the endothelial oscillatory components both increase significantly. The phase coherence between blood flow and temperature changes with the state of vasoconstriction or vasodilation, and increases to a marked extent on heating probably on account of the increased blood flow. It is evident that the static (i.e. tonus) and dynamic (oscillatory) levels of myogenic activity increase and decrease in unison, as 


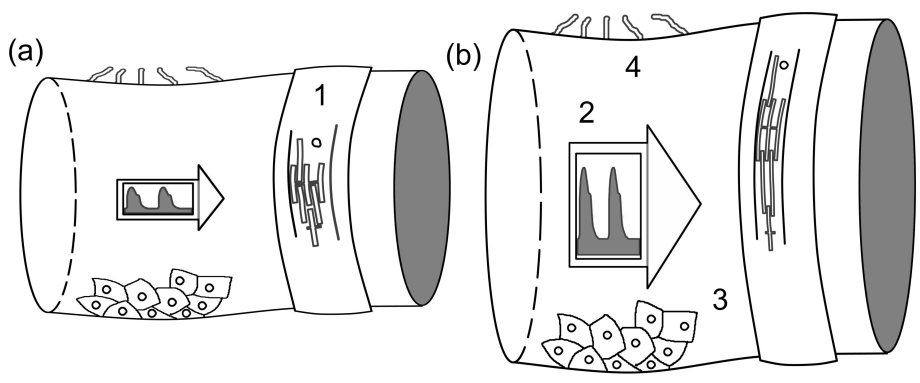

Figure 8. Diagrammatic representation of processes that occur during (a) vasoconstriction and (b) vasodilation. In vasoconstriction, (a) the actin and myosin filaments 1 in the band of smooth muscle around the vessel draw together more closely, reducing the vessel's average radius and resulting in increased myogenic oscillations, a decreased myogenic frequency, and a slightly decreased blood flow. In the vasodilated state (b) the actin and myosin filaments 1 are more separated, corresponding to smaller tonus and larger vessel radius. In addition to the smooth muscles 1 being more relaxed, the amplitude of myogenic oscillations decreases as demonstrated by the results of the present study. At the same time, the vasodilation results in a huge increase in cardiac oscillations as shown in figure 5 and the flow 2 is increased fivefold as shown in figure 2. The vasodilated and vasoconstricted states are also affected by the activity of endothelial cells 3 and sensed by sympathetic nerve fibres 4 connected to the outer walls of the vessels.

summarized in figure 8.

\subsection{Physiological sources of variability}

The physiological sources of variability can be traced back further. The variations in skin blood flow and IHR are found to have significant phase coherence in the myogenic and respiratory frequency intervals. Increased local compliance results in increased phase coherence during heating (an increased 'signal to noise ratio') but endothelial and neurogenic processes that are independent of IHR remain independent when their local amplitude increases. Similarly, despite the pronounced spectral changes in blood flow during cooling, in particular in interval III, the phase coherence between blood flow and IHR did not change.

We suggest that the oscillatory reactivity of vascular endothelium and smooth muscle to the thermal stimuli is associated with the regulation of local vascular resistance, depending on the intensity and direction of temperature change. These oscillatory changes are not associated with increased coupling to the central oscillations of the heart. Rather, they are local oscillatory responses to the thermal perturbation.

\subsection{Vascular responses to cooling and heating}

Earlier work on sequential heating and cooling of the skin (Vuksanović et al. 2008), involving gradual, step-wise, plate-temperature changes within the range $24-42{ }^{\circ} \mathrm{C}$, led to the observation of hysteresis, as shown in figure $9(\mathrm{~b})$. This suggested that different 


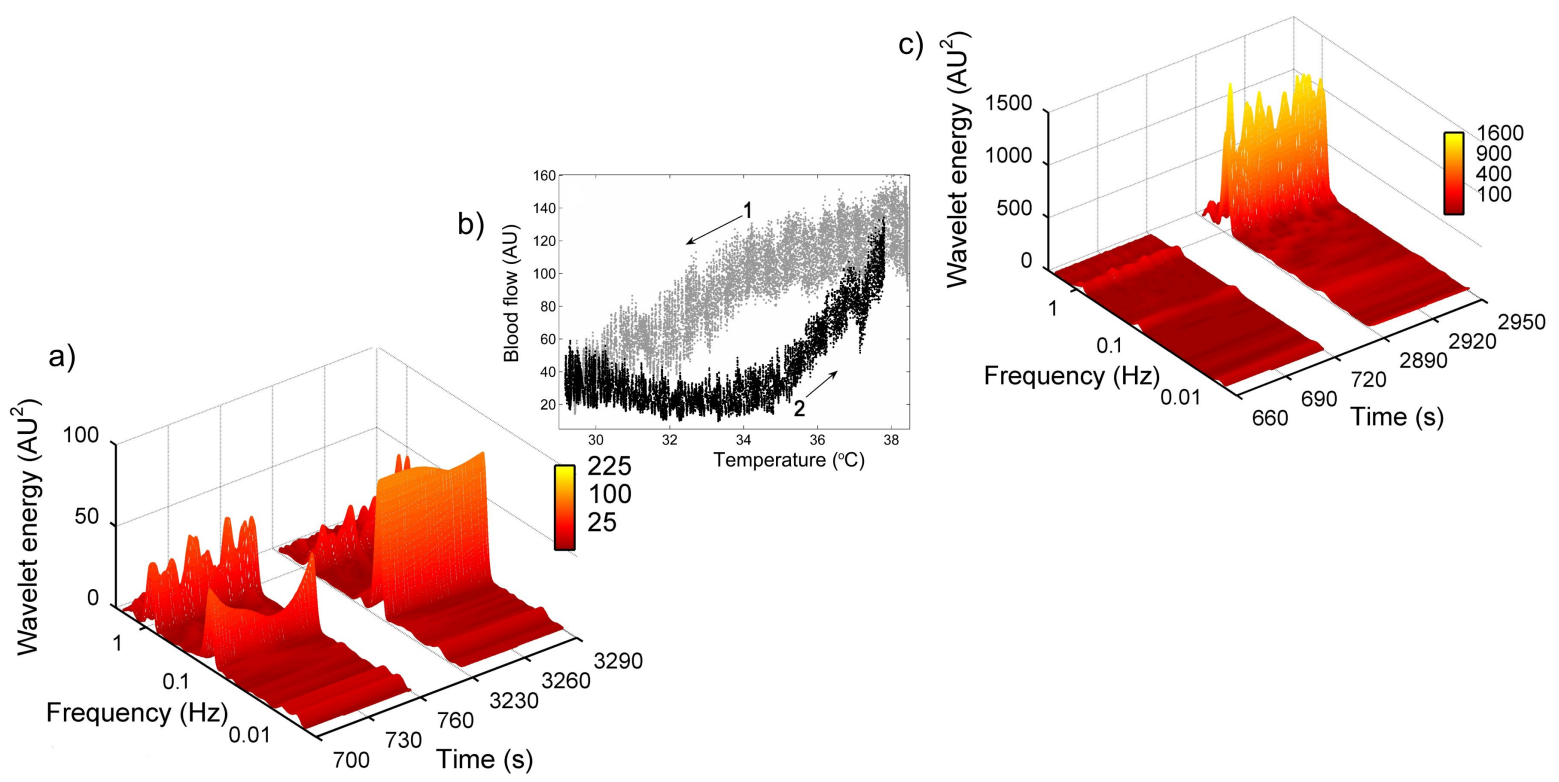

Figure 9. Segments of blood flow wavelet transforms during (a) cooling and (c) heating are compared with segments from the control measurements in each case. The spectral characteristics seen here represent the extremal states of vasodilation and vasoconstriction. (b) The hysteresis curve of skin blood flow and temperature resulting from sequentially heating and cooling the skin.

mechanisms were at work during heating and cooling. Thus, different possible levels of blood flow could coexist at the same temperature. In the present study we have concentrated on the extremal responses to heating and cooling shown by the timefrequency wavelet transforms in figures 9(a) and (c) respectively.

There are at least two possible explanations for the immediate drop in frequency of the myogenic oscillation in skin blood flow on cooling. Either the reduced perfusion slows the metabolic activity in the smooth muscle fibres, slowing their rate of spontaneous oscillation, or the reduced frequency of oscillation is a homeostatic response to cooling which tends to increase the effective vascular resistance and reduces blood flow. The reduced frequency and increased amplitude of the myogenic oscillations is similar to the changes that occur during reduced pressure. A reduced contractile rate is believed to be associated with a reduced stretch response of the smooth muscle cells (Folkow 1964), as observed in rat (Achakri et al. 1995) and rabbit (Oude Vrielink et al. 1990) myogenic oscillations when the tissue is exposed to reduced pressure in vitro. Morris et al. (1996) find increased finger capillary pressure, i.e. reduced precapillary resistance, associated with vasodilation mediated by endothelial nitric oxide synthase, and one vasoconstriction mechanism is reduced nitric oxide synthase activity (Hodges et al. 2006, Yamazaki et al. 2006). During vasodilation the capillary pressure is relatively high, and correspondingly higher precapillary pressure and arteriolar stretch may produce myogenic reflex oscillations at relatively higher frequency. Conversely, during vasoconstriction, relatively low capillary and precapillary pressure may result in lower- 
frequency myogenic oscillations.

The change in the relative proportions of blood flow spectral energy in each band during heating, the reduced proportion of the signal energy at $0.1 \mathrm{~Hz}$, and the increased proportion at the cardiac frequency suggest that, when the smooth muscle fibres are fully extended, their individual oscillations are depressed. The measured changes in spectra are consistent with those found by Geyer et al. (2004), who examined frequencies down to $0.008 \mathrm{~Hz}$ (interval V). We find that activity increases in both the NO-dependent (V) and NO-independent (VI) endothelial spectral intervals.

\subsection{Dynamical relationship between the oscillations in skin blood flow and temperature}

Variability in skin surface temperature is a consequence of variations in the balance between heat supply (from metabolic processes, via the blood) and heat loss (via conduction and, usually, evaporation). Calculation of the wavelet phase coherence of the variabilities in temperature and blood flow for control measurements apparently demonstrates a relationship between them (figure 6), but it is clear that only a proportion of the measured variability in skin temperature is associated with variability in blood flow. This proportion increases significantly when the skin is heated.

The fact that significant phase coherence was not always found in the lowest frequency intervals may be due to the control unit damping low frequency oscillations, or to the small number of cycles at low frequencies limiting our statistical ability to distinguish real from spurious phase relationships. We note that the thermometry was of sufficiently high resolution to identify cardiac and respiratory phase coherence despite the small amplitude of oscillation and the consequent difficulty of detection discussed by Podtaev et al. (2008).

Slow oscillations in skin temperature at about $0.003 \mathrm{~Hz}$ (Nuzzaci et al. 1999), were observed in some signals both before and after temperature change, but these oscillations are at the lower limit that can reliably be probed using the wavelet transform on signals of only 30 min duration, and hence below the lowest frequency interval examined here.

\section{Conclusion}

We have investigated the dynamical aspects of vasoconstriction and vasodilation by use of wavelet and wavelet-phase-coherence analyses in conjunction with a temperaturecontrolled metal plate. In doing so, we have found that vasoconstriction and vasodilation do not just involve changes in the average radii of vessels, as in the traditional picture, but have strong oscillatory components. The dominant frequency is near $0.1 \mathrm{~Hz}$, corresponding to myogenic processes. Cooling produces vasoconstriction, decreased myogenic frequency and increased amplitude on account of the myogenic oscillations becoming more synchronized. The decrease in frequency is probably on account of the vessels being more contracted on average, slowing the vasomotion. Heating produces vasodilation, the tonus decreases, and the myogenic oscillation becomes vanishingly 
small. These changes relax the vessel and enlarge its average radius, thus decreasing vascular resistance and increasing the relative contributions of all the other oscillatory processes. It is clear that the conventional picture of vascular temperature regulation in terms of quasistatic, monotonic, changes in vessel radii and averaged flows cannot be expected to provide an adequate understanding of human temperature control. It must clearly be supplemented by explicit consideration of the oscillatory dynamics.

One advantage of studying vascular activity under thermal perturbation, as described above, is that the differences between the oscillatory dynamics of vasoconstriction and vasodilation can then be illuminated by wavelet analysis. This approach has clearly distinguished between locally and centrally generated oscillations, and it has enabled us to explore the interaction between skin blood flow and temperature. Such studies of the dynamics of vascular contraction/dilation are contributing to our understanding of the characteristic features of vascular thermoregulation, in particular the oscillatory nature of endothelial and myogenic activity. They also represent a possible basis for the non-invasive monitoring of endothelial and myogenic function. In particular, measurements of the changes in local oscillatory processes during thermal challenge could enable a more precise evaluation of microvascular health. For example, under a protocol of cooling and heating, it may be possible to quantify, not just vasoconstriction and vasodilation, but also the specific changes in compliance (amplitude of cardiac oscillation), local myogenic activity and so on.

The endothelial vasodilator response has already been shown to decrease with age. It is also known to be impaired in coronary artery disease, heart failure, diabetes mellitus, hypertension and hypercholesterolemia, as well as in smokers. We may therefore expect that non-invasive measurements under thermal challenge, especially heating, combined with analyses of the kind described in this paper, may in future prove useful for the early diagnosis of a range of pathological conditions and for quantifying the efficacy of treatments that are being prescribed.

\section{Acknowledgements}

We are grateful to Dwain Eckberg for a critical reading of an earlier version of the manuscript and for many very valuable suggestions. We would like to thank Stephen Holt of the Lancaster University Department of Physics' Workshop for his assistance, and the volunteers who contributed to the study. The research was supported by a Royal Society International Short Visit Grant, the Wellcome Trust, the EPSRC, and the EC FP6 project BRACCIA Contract No. 517133 NEST.

\section{References}

Achakri H, Stergiopulos N, Hoogerwerf N, Hayoz D, Brunner H R and Meister J J 1995 Intraluminal pressure modulates the magnitude and the frequency of induced vasomotion in rat arteries J. Vasc. Res. 32 237-246 
Alvarez G E, Zhao K, Kosiba W A and Johnson J M 2006 Relative roles of local and reflex components in cutaneous vasoconstriction during skin cooling in humans $J$. Appl. Physiol. 100 2083-2088

Bandrivskyy A, Bernjak A, McClintock P and Stefanovska A 2004 Wavelet phase coherence analysis: Application to skin temperature and blood flow Cardiovascular Engineering 4 89-93

Bernjak A, Clarkson P B M, McClintock P V E and Stefanovska A 2008 Low-frequency blood flow oscillations in congestive heart failure and after $\beta 1$-blockade treatment Microvasc. Res. 76 224-232

Burton A C and Taylor R M A 1940 A study of the adjustment of periferal vascular tone to the requirements of the regulation of body temperature Am. J.- Physiol. $129565-577$

Camm A J, Malik M, Bigger J T and et al 1996 Heart rate variability - Standards of measurement, physiological interpretation, and clinical use Circulation 93 1043-1065

Eckberg D L 2010 Point: Counterpoint: Respiratory sinus arrhythmia is due to a central mechanism vs. respiratory sinus arrhythmia is due to the baroreflex mechanism $J$. Appl. Physiol. 106 1740-1742

Folkow B 1964 Description of myogenic hypothesis Circ. Res. 15 279-287

Geyer M J, Jan Y K, Brienza D M and Boninger M L 2004 Using wavelet analysis to characteriza the thermoregulatory mechanisms of sacral skin blood flow J. Rehabil. Res. Dev. 41 797-805

Hafner H M, Brauer K, Eichner M, Koch I, Heinle H, Rocken M and Strölin A 2007 Wavelet analysis of skin perfusion in healthy volunteers Microcirculation 14 137-144

Hodges G J, Zhao K, Kosiba W A and Johnson J M 2006 The involvement of nitric oxide in the cutaneous vasoconstrictor response to local cooling in humans J. Physiol.London 574 849-857

Huang A, Sun D and Koller A 2000 Shear stress-induced release of prostaglandin H-2 in arterioles of hypertensive rats Hypertension 35 925-930

Humeau A, Buard B, Mahe G, Chapeau-Blondeau F, Rousseau D and Abraham P 2010 Multifractal analysis of heart rate variability and laser Doppler flowmetry fluctuations: comparison of results from different numerical methods Phys. Med. Biol. 55 62796297

Jamšek J, Stefanovska A and McClintock P V E 2004 Nonlinear cardio-respiratory interactions resolved by time-phase bispectral analysis Phys. Med. Biol. 49 4407-4425

Johnson J M, Yen T C, Zhao K and Kosiba W A 2005 Sympathetic, sensory, and nonneuronal contributions to the cutaneous vasoconstrictor response to local cooling Am. J. Physiol.-Heart Circul. Physiol. 288 H1573-H1579

Kellogg D L 2006 In vivo mechanisms of cutaneous vasodilation and vasoconstriction in humans during thermoregulatory challenges. J. Appl. Physiol. 100 1709-1718 
Kellogg D L, Liu Y, Kosiba I F and O'Donnell D 1999 Role of nitric oxide in the vascular effects of local warming of the skin in humans J. Appl. Physiol. 86 1185-1190

Kitney R I 1974 Analysis and simulation of human thermoregulatory control-system Medical \& Biol. Engin. 12 57-65

Kitney R I 1975 An analysis of the nonlinear behaviour of the human thermal vasomotor control system J. Theor. Biol. 52 231-248

Kitney R I 1980 An analysis of the thermoregulatory influences on heart-rate variability in R I Kitney and O Rompelman, eds., The Study of Heart Rate Variability (Oxford: Clarendon Press) 81-106

Kvandal P, Landsverk S A, Bernjak A, Stefanovska A, Kvernmo H D and Kirkebøen K A 2006 Low frequency oscillations of the laser Doppler perfusion signal in human skin Microvasc. Res. 72 120-127

Kvandal P, Stefanovska A, Veber M, Kvernmo H D and Kirkebøen K A 2003 Regulation of human cutaneous circulation evaluated by laser Doppler flowmetry, iontophoresis, and spectral analysis: importance of nitric oxide and prostaglandines Microvasc. Res. 65 160-171

Leahy M J, de Mul F F, Nilsson G E and Maniewski R 1999 Principles and practice of the laser-Doppler perfusion technique Technol. Health Care 7 143-62

Medow M S, Glover J L and Stewart J M 2008 Nitric oxide and prostaglandin inhibition during acetylcholine-mediated cutaneous vasodilation in humans Microcirculation 15 $569-579$

Minson C T, Berry L T and Joyner M J 2001 Nitric oxide and neurally mediated regulation of skin blood flow during local heating J. Appl. Physiol. 91 1619-1626

Nilsson G E, Tenland T and Öberg P L 1980 Evaluation of a laser Doppler flowmeter for measurement of tissue blood flow IEEE Trans. Biomed. Eng. 27 597-604

Nuzzaci G, Evangelisti A, Righi D, Giannico G and Nuzzaci I 1999 Is there any relationship between cold-induced vasodilatation and vasomotion? Microvasc. Res. $571-7$

Oude Vrielink H H, Slaaf D W, Tangelder G J, Weijmer-Van Velzen S and Reneman R S 1990 Analysis of vasomotion waveform changes during pressure reduction and adenosine application Am. J. Physiol. 258 H29-37

Pawlowski J and Morgan K 1992 Mechanisms of intrinsic tone in ferret vascular smoothmuscle J. Physiol.-London 448 121-132

Podtaev S, Morozov M and Frick P 2008 Wavelet-based correlations of skin temperature and blood flow oscillations Cardiovasc. Eng. 8 185-189

Roustit M, Blaise S, Millet C and Cracowski J L 2010 Reproducibility and methodological issues of skin post-occlusive and thermal hyperemia assessed by singlepoint laser Doppler flowmetry Microvasc. Res. 79 102-108

Shiogai Y, Stefanovska A and McClintock P V E 2010 Nonlinear dynamics of cardiovascular ageing Phys. Rep. 488 51-110 
Shusterman V, Anderson K P and Barnea O 1997 Spontaneous skin temperature oscillations in normal human subjects Am. J. Physiol.: Regulatory Integrative Comp. Physiol. 273 R1173-R1181

Simonet S, Bonhomme E, Fabiani J N and Verbeuren T 2000 Temperature-dependent basal tone in isolated human saphenous veins: implication of TP-receptors Fundam. Clin. Pharmacol. 14 461-467

Smelyanskiy V N, Luchinsky D G, Stefanovska A and McClintock P V E 2005 Inference of a nonlinear stochastic model of the cardiorespiratory interaction Phys. Rev. Lett. 94098101

Stefanovska A 2007 Coupled oscillators: Complex but not complicated cardiovascular and brain interactions IEEE Eng. Med. Bio. Magazine 26 25-29

Stefanovska A and Bračič M 1999 Physics of the human cardiovascular system Contemporary Phys. 40 31-55

Stefanovska A, Bračič M and Kvernmo H D 1999 Wavelet analysis of oscillations in the peripheral blood circulation measured by laser Doppler technique IEEE Trans. Bio. Med. Eng. 46 1230-1239

Thompson-Torgerson C S, Holowatz L A, Flavahan N A and Kenney W L 2007 Coldinduced cutaneous vasoconstriction is mediated by Rho kinase in vivo in human skin Am. J. Physiol.: Heart. Circ. Physiol. $292 \mathrm{H} 1700-\mathrm{H} 1705$

Veber M, Bandrivskyy A, Clarkson P B M, McClintock P V E and Stefanovska A 2004 Wavelet analysis of blood flow dynamics: effect on the individual oscillatory components of iontophoresis with pharmacologically neutral electrolytes Phys. Med. Biol. 49 N111-N117

Vuksanović V, Sheppard L W and Stefanovska A 2008 Nonlinear relationship between level of blood flow and skin temperature for different dynamics of temperature change Biophys. J. 94 L78-L80

Yamazaki F, Sone R, Zhao K, Alvarez G E, Kosiba W A and Johnson J M 2006 Rate dependency and role of nitric oxide in the vascular response to direct cooling in human skin J. Appl. Physiol. 100 42-50 